THU0556

ARTERIAL AND VENOUS THROMBOTIC EVENTS IN IGG4-RELATED DISEASE: A NATIONAL OBSERVATIONAL RETROSPECTIVE STUDY

Blandine Gutierrez ${ }^{1}$, Aurélie Grados ${ }^{2}$, Sylvain Palat ${ }^{1}$, Emmanuel Ribeiro $^{3}$, Noémie Le Gouellec ${ }^{4}$, Julien Haroche ${ }^{5}$, Thomas Papo ${ }^{6}$, Jean-Robert Harlé ${ }^{7}$, Kim Ly ${ }^{1}$, Nicolas Schleinitz ${ }^{7}$, Mikael Ebbo ${ }^{7}$, GEFMAG4 (French IgG4-Related Disease Study Group). 'Limoges University Hospital, Internal Medicine, Limoges, France; ${ }^{2} \mathrm{CH}$ de Niort, Internal Medicine, Niort, France; ${ }^{3} \mathrm{Hôp}$ ital Saint-André, Internal Medicine and Clinical Immunology, Bordeaux, France; ${ }^{4} \mathrm{CH}$ de Valenciennes, Nephrology, Valenciennes, France; ${ }^{5}$ Sorbonne Université, AP-HP, Hôpital PitiéSalpêtrière, Internal Medicine, Paris, France; ${ }^{6}$ Université Paris-Diderot, Internal Medicine, Hôpital Bichat, Paris, France, ${ }^{7}$ Hopital de la Timone, AP-HM, AixMarseille Université, Internal Medicine, Marseille, France

Background: IgG4-related disease (IgG4-RD) is a fibro-inflammatory disorder that can affect virtually every organ. Although arterial involvements have been reported, no studies have examined the occurrence of arterial or venous thrombotic events in these patients.

Objectives: To explore the frequency, the characteristics, and risk factors of arterial and venous thrombotic events in IgG4-related disease patients. Methods: An observational, descriptive, retrospective study was conducted from a multicentric national case registry for IgG4-RD. Patients fulfilled the Comprehensive Diagnostic Criteria (CDC) for IgG4-RD, and all patients with arterial or venous thrombotic events confirmed by imaging during follow-up were analyzed. Clinical, radiological, biological, histological and therapeutic characteristics were retrospectively collected using a standardized online data sheet. Results obtained in patients with thrombosis were compared to those without thrombosis.

Results: One hundred eighty-nine patients with IgG4-RD (135 men/54 women, median age 61 years) were included. During a 12-months median follow-up, one or more arterial thrombotic events occurred in 10 patients and venous thrombotic events in 16 (5.3 and 8.5 events/100 patient-years, respectively).

Arterial complications (coronary artery disease $n=5$, lower limb peripheral arterial disease $n=2$, mesenteric ischemia, transient ischemic attack, and carotid thrombosis: $n=1$ ) occurred on average 30 months [0-140] after the first symptoms of IgG4-RD. They were inaugural in 2 patients without any cardiovascular risk factor, and associated with IgG4-RD arterial involvement in 3 (coronary aneurysms $n=2$, leg arteritis $n=1$ ). Among patients with arterial thrombosis, $60 \%$ had systemic involvement $(\geq 3$ organs involved), $89 \%$ elevated serum IgG4 (> $3 \mathrm{~N}$ in $56 \%$ ), and $57 \%$ CRP $>10 \mathrm{mg} / \mathrm{l}$. Only $5 / 10$ were treated with steroids at the time of arterial complication, and 4 had never been exposed to steroid therapy. Renal involvement was associated with the occurrence of an arterial thrombotic event $(p=0.03)$.

Venous thromboembolic complications (deep venous thrombosis (DVT) $n=12$, pulmonary embolism $n=4$ ) occurred on average 24 months [0-164] after the first symptoms of IgG4-RD, but were inaugural in 6 patients. Usual venous thrombosis risk factors were found in only $3 / 16$. Seven patients had retroperitoneal fibrosis (RPF), 2 had mediastinal fibrosis, $60 \%$ had localized IgG4-RD ( $\leq 2$ organs involved), serum IgG4 level was normal in $67 \%$ and CRP $<10 \mathrm{mg} / \mathrm{l}$ in $79 \%$. Nine patients were on steroids at the time of venous thrombosis. RPF was more frequent in the group of IgG4-RD patients with a venous thrombotic event $(p=0.05)$, and largely associated with DVT in a multivariate analysis $(\mathrm{OR}=8.36$ [2.25-35.93], $p=0.002$ ).

Conclusion: Arterial and venous thrombotic complications are common in IgG4-RD patients. While arterial events are associated with mulitorgan involvement and elevated serum IgG4, venous thrombotic complications appear to affect more likely patients with compressive localized forms of the disease, such as RPF. Mechanisms responsible for this over-risk and clinical benefit of a preventive platelet antiaggregant or anticoagulant treatment in high risk of thrombosis subgroups remain to be evaluated.

Disclosure of Interests: None declared

DOI: 10.1136/annrheumdis-2019-eular.4278

\section{THU0557 NERVE GROWTH FACTOR, SCLEROSTIN AND DKK-1 SERUM LEVELS IN COMPLEX REGIONAL PAIN SYNDROME1 (CRPS-1): A PILOT STUDY ON 41 PATIENTS}

Chiara Crotti ${ }^{1}$, Maria Manara ${ }^{1}$, Francesca Zucchi ${ }^{1}$, Davide Gatti ${ }^{2}$, Maurizio Rossini ${ }^{2}$, Massimo Varenna'. ${ }^{1}$ ASST-Gaetano Pini-CTO, Division of Rheumatology, Milan, Italy, ${ }^{2}$ University of Verona, Department of Medicine, Rheumatology Unit, Verona, Italy

Background: Pain is the hallmark of Complex Regional Pain Syndrome (CRPS). Nerve growth factor (NGF), widely known as pain mediator, is increased in the affected skin $^{1}$ and in tibia bone of rat models of CRPS, while is lowered by administration of anti-NGF antibodies ${ }^{2}$. CRPS usually develops after limb trauma, most frequently a fracture ${ }^{3}$. Some reports considered bone as a main player in CRPS pathogenesis, hypnotizing that sclerostin $(\mathrm{SOST})^{4}$ and Dickkopf-related protein-1 (DKK-1) may be involved in CRPS pathogenesis.

Objectives: To evaluate NGF, SOST, and DKK-1 serum levels from affected arm of CRPS patients and compare them with unaffected one and healthy controls (HCs).

Methods: Adults patients affected by CRPS diagnosed according to IASP criteria at upper limb were consecutively enrolled from April 2017 to August 2018. Patients with prior treatment with bisphosphonates, and history of disorders of mineral metabolism were excluded. Sera from the basilica vein of affected and unaffected arm of CRPS patients were collected, as well as sera from HCs paired for age and sex. NGF, SOST, and DKK-1 concentrations were determined by ELISA kit. Comparisons between patients and HCs were performed by Student test, while comparison between affected and unaffected arms were performed with Wilcoxon test for paired data. Pearson correlation was used to correlate NGF, SOST, and DKK-1 levels with demographic and clinical variables.

Results: The overall population included 41 patients: males (M) $21.9 \%$, mean age at diagnosis $[ \pm$ standard deviation, SD] $61.9 \pm 8.4 \mathrm{yrs}$, median disease duration 67 days (inter quartile range (IQR) 14.0; 22.5), 39 $(95.2 \%)$ experienced a fracture as inciting event, mean VAS pain score (0-100) $54.8 \pm 18.6 \mathrm{~mm}$. Mean NGF levels $(\mathrm{pg} / \mathrm{mL})$ were $12.0 \pm 28.8$ and $11.4 \pm 35.5$ in the affected and unaffected side, respectively, and $13.5 \pm 55.0$ in HCs. NGF was undetectable in most patients; no statistical significant differences of NGF levels were found between patients and HCs. Mean SOST levels (pmol/L) were 32.6 $\pm 16.1,29.8 \pm 17.7$, and $34.0 \pm 13.3$ in affected, unaffected arm, and in $\mathrm{HCs}$, respectively. No statistical significantly differences of SOST levels were found between patients and HCs, while a significant difference was found between affected and unaffected arms $(p=0.03)$. Mean DKK-1 levels (pmol/L) were higher in affected arm $(31.2 \pm 29.5)$ than in unaffected one $(29.3 \pm 28.6)$ or in HCs $(27.5 \pm 18.2)$ without reaching statistical significance. NGF was significantly correlated with VAS pain score $(\mathrm{p}=0.04)$.

Conclusion: To our best knowledge, this is the first study to evaluate NGF, SOST, and DKK-1 levels in adults affected by CRPS-1. SOST levels were significantly higher in affected arms compared to unaffected ones, suggesting a possible role of this bone mediator in CRPS pathogenesis. NGF was consistent with the expression of pain, trough VAS pain score. Further studies need to clarify these preliminary findings.

\section{REFERENCES:}

[1] Li WW, et al. Pain 2010; 151:843-52.

[2] Sabsovitch I, et al. Pain 2008; 138: 47-60.

[3] de Mos M, et al. Pain 2007; 129:12-20.

[4] Robling AG, et al. J Biol Chem 2008;283:5866-75.

Disclosure of Interests: Chiara Crotti: None declared, Maria Manara: None declared, Francesca Zucchi: None declared, Davide Gatti Speakers bureau: Abiogen, Amgen, Janssen-Cilag, Mundipharma, Pfeizer, Maurizio Rossini: None declared, Massimo Varenna: None declared DOI: 10.1136/annrheumdis-2019-eular.4855

\section{THU0558 ADULT-ONSET STILL'S DISEASE PROGNOSIS SCORE. CLINICAL PATTERNS, COMPLICATIONS AND BIOLOGIC TREATMENT}

Ivette Casafont-Solé ${ }^{1}$, Susana Holgado ${ }^{1}$, J. Narváez ${ }^{2}$, Maribel Mora ${ }^{2}$,

Josep Roca ${ }^{1}$, Anahy Brandy-Garcia ${ }^{1}$, Lourdes Mateo ${ }^{1}$, Melania Martínez-Morillo ${ }^{1}$, Laia Gifre ${ }^{1}$, Maria Aparicio Espinar ${ }^{1}$, Águeda Prior-Español ${ }^{1}$, Anne Riveros ${ }^{1}$, Clara Sanguesa $^{1}$, Jordi Camins-Fàbregas ${ }^{1}$, Annika Nack ${ }^{1}$, Joan Miquel Nolla ${ }^{2}$, Alejandro Olive ${ }^{1} .{ }^{1}$ Hospital Universitari Germans Trias i Pujol, Badalona, Spain; ${ }^{2}$ Hospital Universitari de Bellvitge, L'Hospitalet de Llobregat, Spain

Background: Adult-onset Still's disease (AOSD) is an uncommon disease with an unpredictable clinical course and variable prognosis. Sometimes, it requires biologic treatment in early phases. A prognosis score has been described, which has never been applied in a Spanish case series. Objectives: To apply the prognosis score described by Pouchot et al (Systemic Score System (SSS)) on a 64 cases series diagnosed with AOSD in Spanish population and to determine if SSS high values registered at the onset of the pathology are related to AOSD clinical patterns (monocyclic, polycyclic and chronic course), requirement of biologic treatment along the disease's course and development of AOSD clinica 